\title{
Implantable microphone with acoustic tube for fully implantable hearing devices
}

\author{
Eui Sung Jung ${ }^{1}$, Ki Woong Seong ${ }^{2}$, Hyung Gyu Lim ${ }^{1}$, \\ Jyung Hyun Lee ${ }^{3}$, and Jin Ho Cho ${ }^{1,2 a)}$ \\ ${ }^{1}$ Graduate School of Electrical Engineering and Computer Science, Kyungpook \\ National University \\ Sangyuk-dong, Buk-Gu, Daegu, 702-701, S. Korea \\ ${ }^{2}$ Advanced Research Center for Recovery of Human Sensibility, Kyungpook \\ National University \\ Dongin2-Ga, Jung-Gu, Daegu, 700-422, S. Korea \\ ${ }^{3}$ Department of Biomedical Engineering Kyungpook National University Hospital \\ Dongin2-Ga, Jung-Gu, Daegu, 700-422, S. Korea \\ a)jhcho@ee.knu.ac.kr
}

Abstract: Fully Implantable hearing devices consist of a microphone that is implanted under the human skin. However after the implantation, the gain characteristics of the microphone are attenuated at the high frequencies because of the sound filtering effect of the skin and tissue. To solve this problems, we proposed an implantable microphone with an acoustic tube, which generates a resonance effect between the diaphragm and the acoustic transducer inside a case. By performing several experiments in water, it has been confirmed that the frequency response of the implantable microphone at high frequencies can be improved by use of the acoustic tube.

Keywords: implant microphone, acoustic tube, frequency response

Classification: Electronic instrumentation and control

\section{References}

[1] A. E. Deddens, E. P. Wilson, T. H. J. Lesser, and J. M. Fredrickson, "Totally Implantable Hearing Aids: The Effects of Skin Thickness on Microphone Function," American Journal of Otolaryngology, vol. 11, pp. 1-4, 1990.

[2] W. T. Park, K. N. O'Connor, and K. L. Chen, et. al., "Ultraminiature encapsulated accelerometers as a fully implantable sensor for implantable hearing aids," Biomedical Microdevices, vol. 9, June 2007.

[3] E. S. Jung, K. W. Seong, and H. G. Lim, et. al., "Study on frequency response of implantable microphone and vibrating transducer for the gain compensation of implantable middle ear heaing aid," Journal of the Korean Sensors Society, vol. 19, pp. 369-374, 2010.

[4] I. P. Herman, Physics of the Human Body, Springer, Berlin-Heideler-New York, pp. 555-567, 2007. 


\section{Introduction}

Recently, fully implantable hearing aids (FIHAs) have been developed for patients with hearing loss owing to the use of newly developed technologies. To deliver good performance, an FIHA must be able to pick up the desired acoustic sound from a mixture of sound generated by conversations and background noise [1]. However, because the microphone of an FIHA is implanted under the human skin and tissue, the amplitude of the transcutaneous sound wave is attenuated by scattering and absorbing in the skin and tissue. In addition, the frequency response of the microphone is poorer in the acoustic high- frequency band than in the low- to mid- frequency band [3]. Therefore, these drawbacks have an adverse effect on the speech intelligibility of a hearing-impaired person using the FIHA.

In this paper, an implantable microphone that exhibits good sensitivity at high frequencies is proposed. To evaluate of the designed implantable microphone, we measured the sensitivity of several implantable microphones in water using pure-tone stimulation in $0.2-9 \mathrm{kHz}$. On the basis of experimental results thus obtained, it was confirmed that the transfer function of the implantable microphone in the high-frequency band improved because of the resonance effect of the acoustic tube.

\section{Frequency response of implantable microphone}

Figure 1 shows the frequency response of implantable microphones before and after implantation under the skin of a rat [3].

As shown by solid lines, the mean sensitivity of the manufactured implantable microphones was $-35 \mathrm{~dB}$ in a free field (re $0 \mathrm{~dB}=1 \mathrm{~V} / \mathrm{Pa})$. Three weeks after the implantation, the mean sensitivity of the implanted micro-

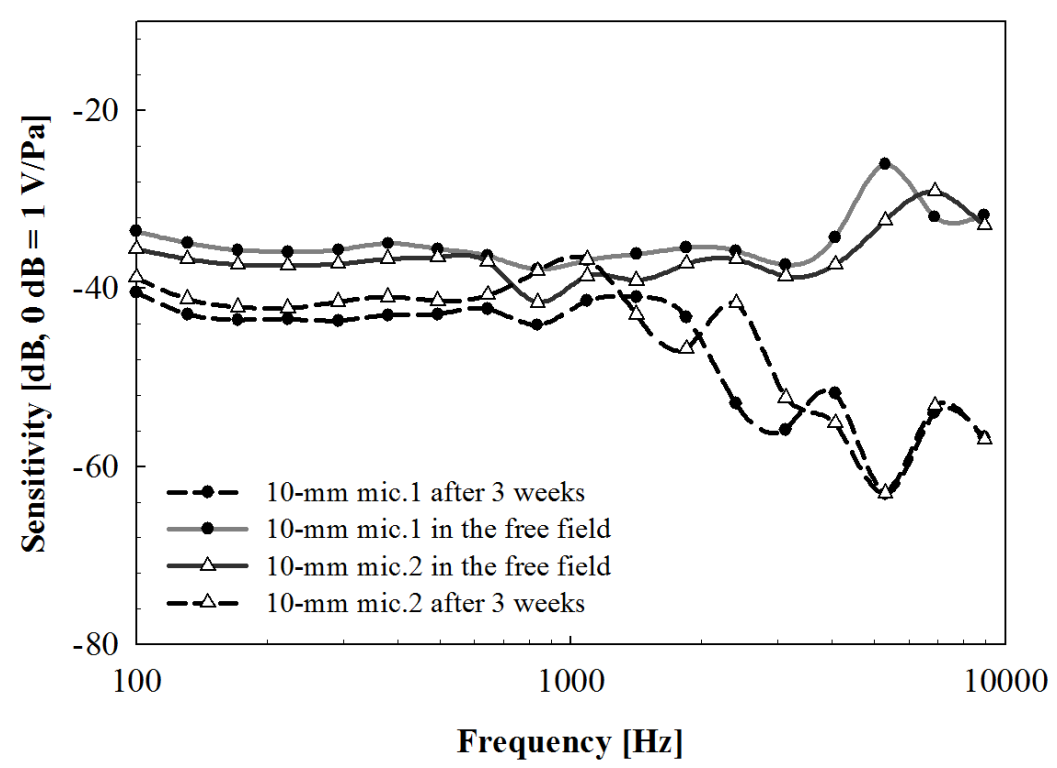

Fig. 1. Frequency response of implantable microphones with 10-mm diaphragm before (solid line) and after (dash line) implantation under the skin of rats. 
phones reduced by $6-8 \mathrm{~dB}$ below $1 \mathrm{kHz}$. The frequency response of the implanted microphones was flat below the cutoff frequency. Above $1 \mathrm{kHz}$, the response had a slope of approximately $-40 \mathrm{~dB}$ per decade. Therefore, the signal-to-noise ratio of the implantable microphone was attenuated because of the reduced frequency response in the high-frequency region.

\section{Implantable microphone with acoustic tube}

The resonance effect produced in the new implantable microphone is similar to that in a closed cylindrical tube. Therefore, the resonance frequency of the spiral-type acoustic tube used in the new implantable microphone can be approximately calculated by using a formula for a cylinder whose one-side end is closed. Moreover, the acoustic tube produces resonant waves at $\lambda / 4$, $3 \lambda / 4$, and so on, and hence, the high frequency of acoustic sound is amplified.

The resonance frequency of a closed tube, $f$, is

$$
f=n v /[4(L+0.4 d)]
$$

where " $n$ " is an odd integer $(1,3,5 \ldots) . L$ is the length of the tube, $v$ is the sound speed in air, and $d$ is the diameter of the acoustic tube. This closed tube produces only odd harmonics, and its fundamental frequency is half that of an open cylinder. Figure 2 shows the structure and a photograph of the proposed implantable microphone with an acoustic tube. The new implantable microphone unit consists of an electret condenser microphone (ECM) as an acoustic transducer, a case with a spiral-type acoustic tube, a 13-mm titanium diaphragm with a thickness of $20-\mu \mathrm{m}$, an o-ring, and a cap for fixing the diaphragm. The case and the diaphragm must be made of biocompatible materials. However, experimental implantable microphones were made of commercial grade titanium to reduce cost, and to make it easier to perform in-vitro experiments in water.

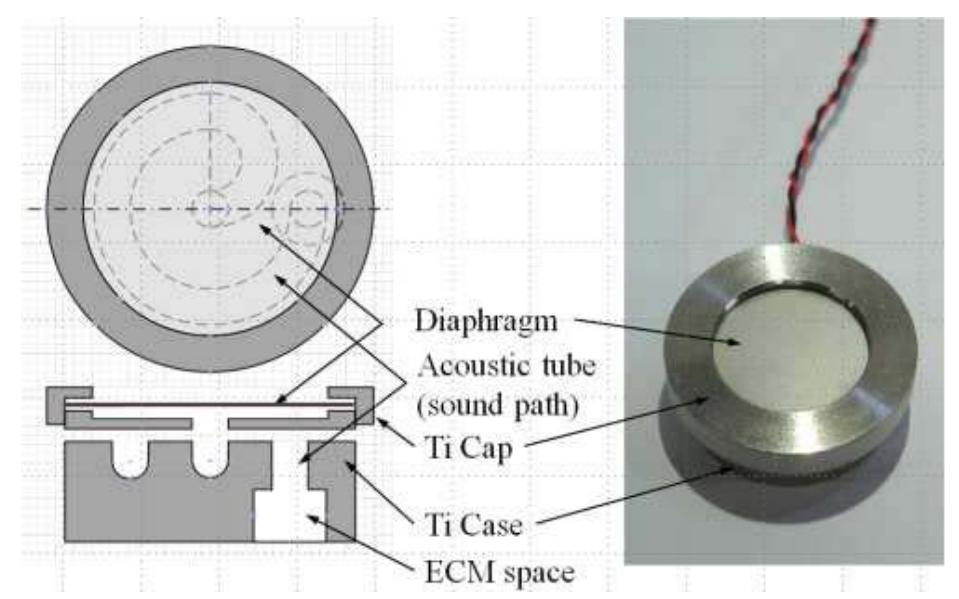

Fig. 2. Design and photograph of implantable microphone. 


\section{Experimental results and discussion}

The frequency response of a microphone prototype was experimentally measured to verify the change in the compensated sensitivity with the length of the acoustic tube in water. In-vitro experiments were performed in water in an environment similar to that of human skin and tissue [4].

All the experimental equipment used for generating sound and for saving the data were interfaced with a PC and controlled automatically by using the LabVIEW program. We measured the sensitivity of several microphones in water and air using a pure sound stimulus with a sound pressure level of $94 \mathrm{~dB}$ at $0.1-8 \mathrm{kHz}$. The sound was transmitted by an omnisource loudspeaker (Bruel \& Kjaer, 4295) placed $1 \mathrm{~m}$ in front of the implantable microphone. The transmitted sound pressure level was measured using a probe-tube microphone (Etymotic Research, ER-7C) placed $1 \mathrm{~mm}$ in front of the water surface and recorded using a data measurement board (NI Co., PCI-6251).

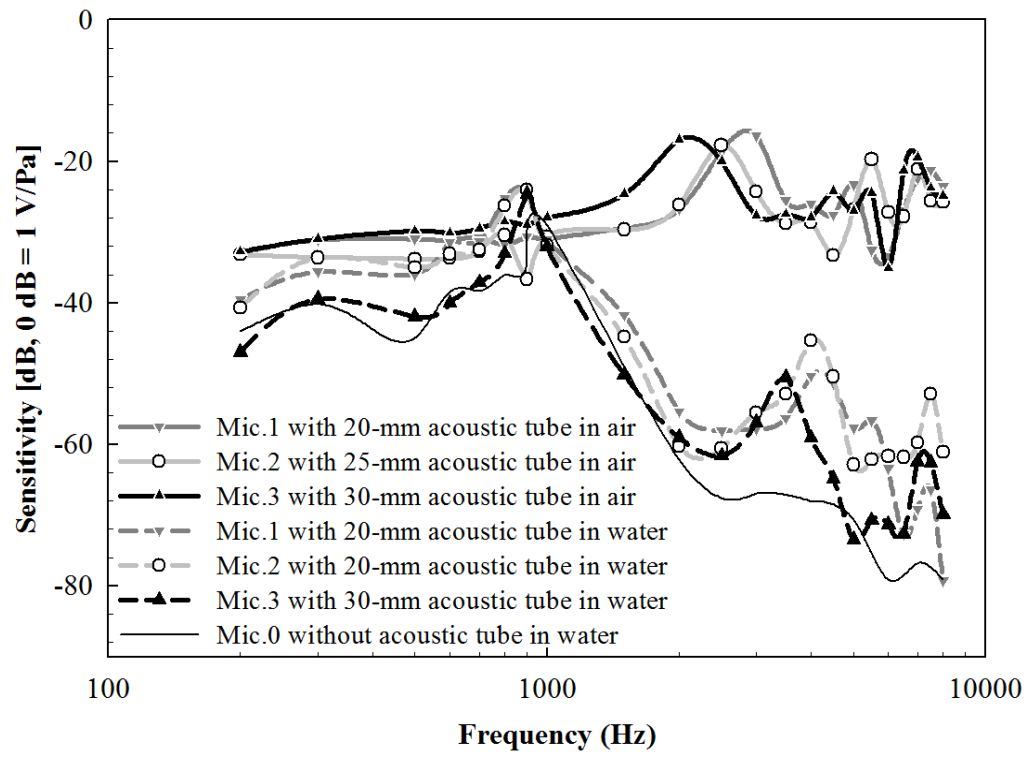

Fig. 3. Frequency response of implantable microphones as each of an acoustic tube with length of $20 \mathrm{~mm}$ (inverse triangle line), $25 \mathrm{~mm}$ (triangle line), $30 \mathrm{~mm}$ (open circle line), and without an acoustic tube (fine solid line) in air and water.

Figure 3 shows the measured frequency response for each of the three implantable microphones with an acoustic tube and for the normal implantable microphone in air and water. The graph shape for the implantable microphones was flat in the low-frequency region and the mean sensitivity was $-40 \mathrm{~dB}$ and. In addition, the frequency response showed the characteristic of second-order low-pass filter with $700-800 \mathrm{~Hz}$ resonance frequencies. The frequency response of the microphone with the 20-mm acoustic tube exhibited considerable improvement at $3.4 \mathrm{kHz}$, as shown by the triangle broken line. Similar improvements were observed for the 25-, and 30-mm acoustic tubes 
at 4 and $4.5 \mathrm{kHz}$, as shown by the circle and reverse triangle broken lines, respectively. At the resonance frequencies by the acoustic tube, the frequency response of the fabricated implantable microphones is about $20 \mathrm{~dB}$ higher than that of the normal implantable microphone (fine black line). Therefore, the resonance effect of the acoustic tube improved the effective dynamic range of the implantable microphones at high frequencies. As lengths of acoustic tube of the microphone, it can be adjusted to the resonance frequency for the compensated the sensitivity

\section{Conclusion}

This paper presents a new implantable microphone with an acoustic tube that can compensate for the distortion produced by the sound filtering effect of the skin and tissue at high frequencies of over $1 \mathrm{kHz}$. By varying the length of the acoustic tube, it was adjusted to the second resonance frequency in the high frequency range. Owing to the resonance effect of the acoustic tubes, the sensitivity of the implantable microphones with an acoustic tube was approximately $20 \mathrm{~dB}$ greater than that of the microphones without an acoustic tube at frequencies between 3.5 and $5 \mathrm{kHz}$. This increase in the sensitivity resulted in an increase in the dynamic frequency range of the new implantable microphones.

\section{Acknowledgments}

This work was supported by the Ministry of Health \& Welfare (A092106), and by MEST (No. 2010-0010570) in KOREA. 